\title{
IgA NEPHROPATHY IN A PATIENT WITH SYSTEMIC LUPUS ERYTHEMATOSUS
}

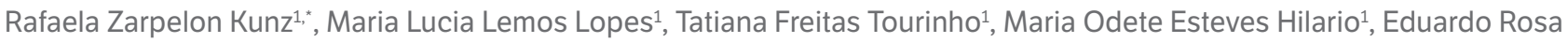
de Oliveira ${ }^{1}$, Thiago Willers ${ }^{1}$, Rafael Coradin ${ }^{1}$, Gilberto Scanagatta ${ }^{1}$, Raissa Velasques de Figueiredo ${ }^{1}$, Gabriela Sasso Padilha ${ }^{1}$, Bruno Trevisan ${ }^{1}$, Luana Ribeiro Carlos ${ }^{1}$

1. Universidade Federal de Ciências da Saúde de Porto Alegre, Porto Alegre (RS), Brazil.

*Corresponding author: consultoriowillers@gmail.com

\section{BACKGROUND}

Systemic lupus erythematosus (SLE) is an autoimmune disease of multisystemic involvement and one of the most striking manifestations is nephritis. The classic clinical presentation is persistent proteinuria and/or cell cylinders or active urine sediment, that is, five or more red blood cells or leukocytes per high-magnitude field. IgA nephropathy, on the other hand, is manifested by persistent microscopic or sporadic macroscopic hematuria. High levels of anti-DNA and complement consumption may point to kidney activity related to lupus. In this report, we present the case of a patient with systemic lupus erythematosus with previous involvement of class IV lupus nephritis and who later develops IgA nephropathy.

\section{CASE REPORT}

Female patient, 40 years old, with systemic lupus erythematosus diagnosed in 1998, who met the criteria for cutaneous involvement, reactive antinuclear factor (ANA), alopecia and lupus nephritis class IV in a biopsy performed in 1999, in need of pulse therapy with methylprednisolone and after with cyclophosphamide in the diagnosis. She had no other comorbidities. Currently using hydroxychloroquine $400 \mathrm{mg}$, azathioprine $150 \mathrm{mg}$, enalapril $10 \mathrm{mg}$ and prednisone $10 \mathrm{mg}$. During outpatient follow-up, persistent microscopic hematuria was observed, but without laboratory alterations compatible with the disease activity - complement and normal inflammatory tests, nonreactive anti-DNA. Common urine examination reveals the presence of hemoglobin and many erythrocytes, with absence of cell cylinders and proteinuria. Research on urinary erythrocyte dysmorphism was carried out, which showed changes in $52 \%$ of red blood cells. Urinary tract ultrasound showed no changes and preserved renal function. We opted for a new renal biopsy in 2019, and a pathological study compatible with IgA nephropathy, mesangial proliferative. Mesangial granular IgA $(++)$ immunofluorescence, mesangial granular IgM (++), mesangial granular $\mathrm{C} 3 \mathrm{C}(++)$. New pulse therapy with methylprednisolone was performed for three days.

\section{CONCLUSION}

Even though SLE can coexist with other autoimmune diseases, it is rarely described in association with nephropathies of nonlupus etiology. The importance of distinguishing between SLE kidney disease activity and non-lupus nephropathies mainly implies renal prognosis and the adoption of therapeutic measures. 